\title{
ADVANCED BREAST METAPLASTIC CARCINOMA IN A YOUNG PATIENT: A CASE REPORT
}

Mirella Laranjeira Nunes¹, Carlos Eduardo Caiado Anunciação², Vidianna Barbosa Sampaio², Rossano Robério Fernandes Araújo², Cinthya Roberta Santos de Jesus², Ana Leide Guerra dos Santos², Bruno Pacheco Pereira², João Esberard de Vasconcelos Beltrão Neto²

${ }^{1}$ Universidade de Pernambuco - Recife (PE), Brazil.

2Department of Mastology, Oswaldo Cruz University Hospital - Recife (PE), Brazil.

Objective: The aim was to evaluate an atypical case due to the rare pathologic finding of metaplastic breast carcinoma in a young woman, with an aggressive pattern. Case Report: A 45-year-old female presented with suspicious palpable right axillary lesion at level 1 topography of $2.5 \mathrm{~cm}$ size on the physical examination. No breast mass was palpable. Mammography was BIRADS classification 1. Breast and axillar ultrasound done 2 months before showed benign findings on the left side and axillary lymph node (LN) of $2.3 \mathrm{~cm}$ and breast nodule of $1.1 \times 0.9 \mathrm{~cm}$ on the right side. Core-needle biopsy showed fibroadenoma in the right-sided breast nodule and metastatic carcinoma in the axillary LN. Immunohistochemistry expression of the markers was consistent with breast origin and was progesterone and estrogen receptors positive and HER-2 negative. Magnetic resonance imaging (MRI) showed this atypical LN with $1.5 \mathrm{~cm}$. Clinical staging is T0N1M0. Neoadjuvant chemotherapy was performed with Adriamycin, cyclophosphamide, and paclitaxel. There was tumor remission with another MRI and ultrasonography showed the node metastasis with $1 \mathrm{~cm}$. Right radical mastectomy was performed. Anatomopathology showed cytoarchitectural changes due to chemotherapy, complete pathological response in the LN, and immunohistochemistry unchanged. In addition, tumorectomy were performed in the left-sided nodule, with anatomopathology showing ductal ectasia and histiocitary abscess. Radiotherapy at the supraclavicular area and tangents was performed and tamoxifen was prescribed. The patient remained cancer free for 2 years after surgery. Conclusion: Metaplastic carcinoma represents less than $1 \%$ of breast cancers. Histologically, it is invasive and has subtypes based on the proportion of squamous, mesenchymal, and heterologous elements, such as cartilage and bone. Most common in women in the fifth decade and rare in younger than 35 years old. Usually, it is triple negative and has hematogenous dissemination, with little LN involvement and more distant metastasis. There is currently no described standard treatment. Owing to aggressiveness and poor prognosis, chemotherapy and modified radical mastectomy are performed. It is often refractory to standard regimens, so chemotherapy is indicated through the extrapolation of current data to invasive ductal carcinoma. The metastasis rate is about $35 \%$ in 5 years. The main prognostic factor is the size of the tumor at the time of diagnosis, LN metastasis, and poorly differentiated tumors.

Keywords: Breast Neoplasms; Neoplasm Metastasis. 